Pacific Journal of Mathematics

INFINITESIMAL MOTIONS OF A BIPARTITE FRAMEWORK 


\title{
INFINITESIMAL MOTIONS OF A BIPARTITE FRAMEWORK
}

\author{
WALTER Whiteley
}

\begin{abstract}
A recently established criterion for stresses on any bar and joint framework with a complete bipartite graph is converted into explicit criteria for infinitesimal motions of the framework. These criteria, based on quadric surfaces through the joints, include and complete those developed in recent years in the study of geodesy with range satellite networks. The infinitesimal motions are displayed in a simple geometric form, appropriate to any dimension. This geometric description is used to establish necessary and sufficient conditions, based on ruled quadric surfaces, for which bars may be added to a bipartite framework without removing a prior infinitesimal motion. These criteria are applied to the behaviour of structural engineering space frames.
\end{abstract}

1. Introduction. In a recent paper, Bolker and Roth presented an analysis of static stresses in any bar and joint framework with an underlying bipartite graph [1]. In spite of their title ("When is a bipartite graph a rigid framework?"), the paper gave explicit criteria only for the number of stresses in the framework, and left implicit the number of infinitesimal (or finite) motions which resulted.

Our work began with an observation that their static criterion yielded explicit criteria (both necessary and sufficient) for the presence of nontrivial infinitesimal motions: essentially that all joints of the framework lie on a quadric surface (\$2) [7]. This simple geometric conclusion suggested that the motions must flow in some natural way from the quadric surface and we present two descriptions of the motion drawn from the quadric surface (§3). One description is informal and geometric, the other is more detailed and analytic though still geometric. We feel that the general geometric approach gives a stronger intuition for the basic connections, but the analytic approach is easier to condense, and easier to use in detailed form later in the paper.

Once we "see" the source of the motions we can pose, and answer, an interesting question: What bars can be added to a bipartite framework without blocking the infinitesimal motion? (§4). When additional bars are added to a bipartite framework, the essential condition for an infinitesimal motion is a ruled quadric surface through all the joints, containing the lines of all added bars (Theorem 3). Ironically, while Bolker and Roth 
asked for efforts to make their work into an applicable theory, portions of their results, in dimensions 2 and 3, had been anticipated by applied work in geodesy and range satellite networks $[5,10,11,12]$. In this setting, the problem was: given $m$ satellites in space, and $n$ points on earth, we measure (with lasers or electronically) the $m n$ distances between earth stations and satellites. Can we now calculate the exact distances between the points on earth? If we know some distances among the satellites, can we calculate the exact distances between the points on earth? Work in this area, over the last two decades, had given the explicit criteria for most cases when these calculations had singularities (equivalently, infinitesimal motions) $[5,11]$, and had examined examples of finite mechanisms $[5,10$, 12, 13]. Provided that the points on earth were not "near" such a singularity, and that the distances on earth were known roughly (within an error smaller than the distance to the singularity), then the calculations will yield an accurate answer for the distances on earth.

We discovered this prior work while doing the final writing of this paper. In retrospect we can say that the static analysis of Bolker and Roth offered a single unified attack for all dimensions, yielding exact necessary and sufficient conditions for infinitesimal (or static) rigidity of a complete bipartite framework - results which clearly extend the results developed in the earlier kinematic work in geodesy.

Our work builds on this completeness, and differs in approach from the previous work on infinitesimal motions. Instead of detailed calculations and exacting formulae, we use a direct geometric approach to trace the motions and pursue the consequences for bipartite frameworks with added bars. There always are advantages in having several methods available for attacking a problem. We offer this geometric vision in the belief that it simplifies and completes the previous work by giving a direct insight into the patterns of the motions - patterns not available in any of the previous work.

This geometric insight can also be applied to unify and extend work on the question: When do the infinitesimal motions extend to finite motions? In this area the work dates back to the turn of the century [4], and it seems preferable to present the results in a separate paper.

Our initial motivation for studying the infinitesimal motions and infinitesimal rigidity of frameworks lay in work on the stuctural engineering of frameworks. In this spirit we close our paper with an application of Theorem 3 to the behavior of standard space-frames built by engineers (§5). It is a delightful bonus to find this pretty geometry opening a direct insight into some of the most common, but poorly understood, engineering structures [2]. 
Our debt to the paper of Bolker and Roth is obvious, and we have benefited from additional communications with both of them. Less obvious, but as important, is our debt to the other members of the Structural Topology Research Group at Université de Montréal. The entire setting for our work comes from the research of this group into applications of geometry to problems in architecture and structural engineering, and our results have been refined within discussions with the group, in particular with Henry Crapo and Janos Baracs.

The study of bipartite frameworks has flowed from the early, kinematic work in geodesy, to the static results in Bolker and Roth and back to our kinematic reevaluation of their results. In light of our own recent propaganda and polemics for the value of statics [3], we appreciate the irony of this kinematic reassertion. The flow back and forth between these techniques is an underlying theme of a number of recent papers, and as this paper illustrates, this flow is aided by a solidly geometric view of both topics. We look forward to the possibility of continued cross-fertilization between structural engineering, geodesy, and a projective geometric theory of infinitesimal motions and statics for frameworks.

2. When does a bipartite framework move? We will follow the basic notation of Bolker and Roth [1]. However hidden under this Euclidean facade will be intuitions and processes which come from the projective theory of mechanics $[3,8]$. For simplicity most of the examples we describe are in 3-space, but many of the diagrams show plane-figures, sections of the larger frameworks, and some of the theorems are stated in $d$-dimensional form.

We briefly recall the necessary vocabulary. A framework in $\mathbf{R}^{d}$ is an indexed set $V$ of points $\left(p_{1}, \ldots, p_{v}\right)$ in $\mathbf{R}^{d}$ and a set of bars $E$ of unordered pairs $\{i, j\}, 1 \leq i, j \leq v$. For simplicity we assume that $p_{i} \neq p_{j}$ when $\{i, j\} \in E$. We speak of the framework as a realization in $\mathbf{R}^{d}$ of an underlying graph with the vertex set $\{1, \ldots, v\}$ and edge set $E$.

An infinitesimal motion of a framework with joints $V$ and bars $E$ is an assignment of vector velocities $v_{i}$ to the joints $p_{i}$ such that the length of the bar $\left|p_{i}-p_{J}\right|$ is constant for all bars. By a simple differentiation, this condition is replaced by

$$
\left(v_{i}-v_{J}\right) \circ\left(p_{i}-p_{j}\right)=0 \text { for all }\{i, j\} \in E .
$$

Every framework has some infinitesimal motions - those corresponding to the rigid motions of the space (translations, rotations, etc.) [1, §4]. If all the infinitesimal motions of a framework correspond to rigid 
motions of the space we say the framework is infinitesimally rigid. Otherwise we say there is an internal motion. In particular, if $\left(v_{m}-v_{n}\right) \circ\left(p_{m}-\right.$ $\left.p_{n}\right) \neq 0$ for some pair of joints (not a bar) then the motion is an internal motion, and if the joints of the framework span the space, in an affine sense, every internal motion will have such a pair of spreading (or approaching) joints.

The infinitesimal motions form a vector space, and if the joints span (in an affine sense) at least a hyperplane of the space $\mathbf{R}^{d}$, the rigid motions induce a subspace which has dimension $\frac{1}{2} d(d+1)$. As a convention, the difference between the dimension of the space of infinitesimal motions, and of the space of rigid motions is called the degree of internal freedom. (When we say a set of points spans a space, this will always be in the affine or projective sense.)

Finally, a bipartite framework is a framework whose underlying graph is a complete bipartite graph: the vertices can be divided into two disjoint sets $A, B$ such that the edges are all unordered pairs $\{i, j\}$ with $i \in A$ and $j \in B$. Writing $|A|=m$ and $|B|=n$ we speak of the bipartite graph $K_{m, n}$. As a slight abuse of terminology we will write $A$ and $B$ to denote both the sets of vertices of the graph and the corresponding joints in the realization.

In [5, p. 69] and again in [1, Theorem 15], it is shown that a framework in 3-space, realizing the graph $K_{4,6}$ has an internal infinitesimal motion iff the joints all lie on a quadric surface, or the 4 points lie in a plane. Our first task is to generalize this result to arbitrary bipartite frameworks and all dimensions. The entire result is just a careful translation into kinematic terms of the static Theorem 10 of [1].

THEOREM 1. A bipartite framework realizing the bipartite graph $K_{m, n}$ $(m, n \geq 2)$ in $\mathbf{R}^{d}(d>1)$ has a nontrivial infinitesimal motion iff either

(i) the joints of $A \cup B$ lie on a quadric surface, or

(ii) one side ( $A$ or $B$ ) lies on a hyperplane along with at least one joint of the other side, or

(iii) one side ( $A$ or $B$ ) lies on a hyperplane $H$ and lies on a quadric surface within the hyperplane.

Proof. [1, Theorem 10] records the dimension of the space of stresses, a number which we will call $w$. If $\bar{A}$ is the affine span of $A$, etc., $C=(\bar{A} \cap B) \cup(\bar{B} \cap A), k=|C|, h=\operatorname{dim}(\bar{C})$ and $Q$ is the space of quadric surfaces in $\bar{C}$ which contain all joints of $C$, then Theorem 10 says

$$
\begin{aligned}
w= & (|A|-\operatorname{dim}|\bar{A}|-1)(|B|-\operatorname{dim}|\bar{B}|-1) \\
& +k+\operatorname{dim} Q-\frac{1}{2}(h+1)(h+2)
\end{aligned}
$$


(if $h=0$ then $\operatorname{dim} Q$ is defined to be 0 , but if $k=0$ then $\operatorname{dim} Q=1$ ).

To use this count of stresses, we need the fundamental equivalence between statics and infinitesimal mechanics (a restatement of the linear algebra theorem that the row rank = column rank for the rigidity matrix) $[1, \S 4]$ :

$$
|E|-\operatorname{dim}(\text { Stress space })=|V| \cdot d-\operatorname{dim}(\text { Motion space }) .
$$

We now assume we have a bipartite framework such that the points have a space of rigid motions of $\operatorname{dim}=j$, and let $F$ denote the degree of internal freedom:

$$
m \circ n-w=(m+n) d-(F+j) .
$$

We replace $w$ by the expression given above and solve for $F$ :

$$
\begin{aligned}
F= & m(d-\operatorname{dim}|\bar{B}|-1)+n(d-\operatorname{dim}|\bar{A}|-1)+k+\operatorname{dim} Q \\
& +(\operatorname{dim}|\bar{A}|+1)(\operatorname{dim}|\bar{B}|+1)-\frac{1}{2}(2 j+(h+1)(h+2)) .
\end{aligned}
$$

The rest of the proof is by cases, depending on $\operatorname{dim}|\bar{A}|$ and $\operatorname{dim}|\bar{B}|$.

Case 1. $A$ and $B$ both span the space.

Then $\operatorname{dim}|\bar{A}|=d, \operatorname{dim}|\bar{B}|=d, j=\frac{1}{2} d(d+1), C=A \cup B, k=m+$ $n$ and $h=d$.

Equation (1) reduces to

$$
\begin{aligned}
F= & m(-1)+n(-1)+m+n+Q(A \cup B)+(d+1)^{2} \\
& -\frac{1}{2}(d(d+1)+(d+1)(d+2))=Q(A \cup B) .
\end{aligned}
$$

We conclude that the framework has a motion iff the joints lie on a quadric surface.

Case 2. $A$ and $B$ are both flat, spanning two hyperplanes. Thus $\operatorname{dim}|\bar{A}|=d-1, \operatorname{dim}|\bar{B}|=d-1, j=\frac{1}{2} d(d+1)$. We ignore $C$, letting the nonnegative number $k+\operatorname{dim} Q-\frac{1}{2}(h+1)(h+2)$ disappear.

$$
F \geq m \circ 0+n \circ 0+d^{2}-\frac{1}{2} d(d+1)=\frac{1}{2} d(d-1)>0 .
$$

We always have both an internal motion and a degenerate quadric surface of two hyperplanes.

Case 3. $A$ spans a hyperplane and $B$ spans space. Thus,

$$
\operatorname{dim}|\bar{A}|=d-1, \operatorname{dim}|\bar{B}|=d, j=\frac{1}{2} d(d+1), C=A \cup(\bar{A} \cap B),
$$

and $h=d-1$. 
Equation (1) becomes

$$
\begin{aligned}
F= & m(-1)+n \circ 0+m+|\bar{A} \cap B|+\operatorname{dim} Q(C)+d(d+1) \\
& -\frac{1}{2}(d(d+1)+d(d+1)) \\
= & |\bar{A} \cap B|+\operatorname{dim} Q(C) .
\end{aligned}
$$

$F>0$ iff either $|\vec{A} \cap B|>0$ and some joint of $b$ lies in the hyperplane of $A$, or $|\bar{A} \cap B|=0$ and $\operatorname{dim} Q(A)>0$, in which case the joints of $A$ lie on a quadric surface of the hyperplane.

Case 4. $A$ or $B$ do not even span a hyperplane.

If possible we choose an "axis" (a space of $\operatorname{dim} d-2$ ) through the flat side (say $A$ ) leaving at least two joints of $B$ off the axis. When we freeze the axis and one of these $\left(b_{i}\right)$, the second $\left(b_{j}\right)$ will rotate about the axis, giving an internal motion (Figure 1A). There is a motion and the every flat side lies on a degenerate quadric surface of any hyperplane through this side.
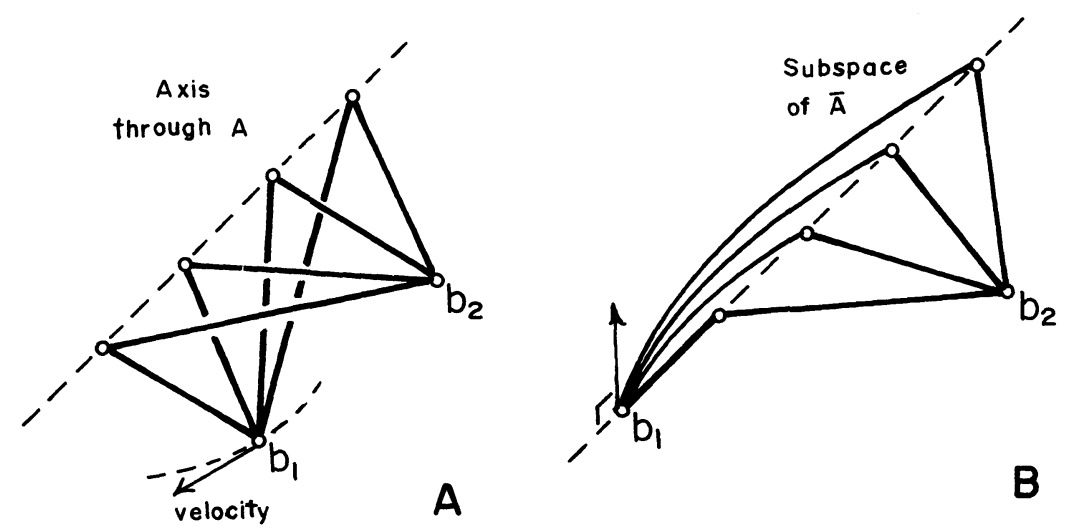

\section{FIGURE 1}

If such an axis was impossible then at least one $b_{j}$ lies in $\bar{A}$ (since $n \geq 2$ ). We now freeze all other joints and give this $b_{j}$ a velocity normal to $\bar{A}$. This is an internal motion since any rigid motion freezing $A$ will freeze $\bar{A}$ (Figure 1B). Once more we have the motion required by part (ii) (and part (iii)) of the statement.

If we ignore the special degeneracies of part (ii), then the sense of the theorem is summarized in the following sufficient condition for a motion. For the plane and 3-space, this result appeared in $[5$, p. 71] and [10, Satz 2; 11, Satz 11]. 
COROLlary 1.1 Any bipartite framework (with more than 2 joints) realized with all its joints on a quadric surface in $\mathbf{R}^{d}(d>1)$ will have an internal motion.

Proof. If $A$ and $B$ span the space, then Theorem 1 (i) applies. If $A$ lies in a hyperplane then the quadric surface gives the required quadric within the hyperplane for Theorem 1 (iii).

In the case of frameworks in 3-space, the kind engineers actually build and geodesists measure, we would like to be more precise about the number of internal motions. We offer some specific corollaries to the proof, which we will need later in the paper. A form of Corollaries 1.2 and 1.4 is contained implicitly in [11]. If desired, we could read off the corresponding results in any dimension.

COROLlARY 1.2 Given a bipartite framework in 3-space, with $A$ and $B$ both spanning the space, the degree of internal freedom equals the dimension of the space of quadratic surfaces through all the joints.

COROLlaRy 1.3 Given a bipartite framework in 3-space with A spanning one plane and $B$ spanning a second plane, then the degree of internal freedom is $\geq 3$, and is exactly 3 when no joints lie on both planes.

Proof. We have $\operatorname{dim}|\bar{A}|=2, \operatorname{dim}|\bar{B}|=2, j=6, d=3$. If there are $k$ joints on the common line, equation (1) gives:

$$
F=3+k+\operatorname{dim} Q(C)-\frac{1}{2}(h+1)(h+2) \geq 3 \text {. }
$$

REMARK. When $k$ joints lie on the common line then simple calculations show that one degree of internal freedom is added for each joint on the line but one of the original three degrees is removed for each of the first three distinct points occupied on this line. In a sense these joints absorb the original motions, turning them into a set of simple vibrations, each normal to the plane which must contain all bars into a joint on the common line. This observation offers a foretaste of Theorem 3 (ii).

COROLlaRY 1.4 Given a bipartite framework in 3-space, with $A$ spanning a plane and $B$ spanning space, and $B^{\prime}=\bar{A} \cap B, q\left(A \cup B^{\prime}\right)=$ the dimension of the space of conics through $A \cup B^{\prime}$ in the plane of $A$, then the degree of internal freedom $=\left|\bar{B}^{\prime}\right|+q\left(A \cup B^{\prime}\right)$.

COROLlary 1.5 If $A$ is collinear then there are at least $|B|-1$ degrees of internal freedom. 
3. How does a bipartite framework move? Corollary 1.1 predicts an internal motion for a bipartite framework with its joints on a quadric surface. What geometric or algebraic characteristic of quadrics is expressed in this motion? A simple example in space will take us to the core of the explanation.

EXAMPLE 1. The sphere. We take an arbitrary $K_{m, n}$ with all of its joints on a sphere in 3-space, with the equation

$$
x^{2}+y^{2}+z^{2}=r^{2} \quad \text { or } X \circ X=r^{2}
$$

Each joint in $A$ is assigned a velocity radially outward, $u=a$.

Each joint in $B$ is assigned a velocity radially inward, $v=-b$.
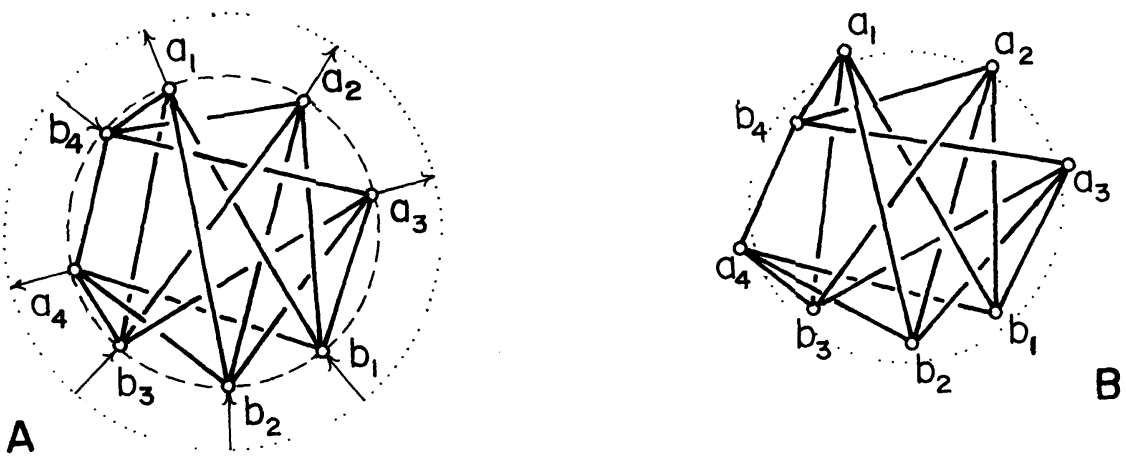

Figure 2

These velocities preserve the length of the bar $a b$ because

(i) geometrically, the chord $a b$ makes equal angles with the two radial vectors (Figure 2) and

(ii) algebraically, $(a-b) \circ(u-v)=(a-b) \circ(a+b)=a \circ a-$ $b \circ b=0$.

This result holds for any pair of points $a$ and $b$ on the sphere, so the assignment is an infinitesimal motion. It is not a rigid motion since for any distinct points $a, a^{\prime}$ on the sphere

$$
\left(a-a^{\prime}\right) \circ\left(u-u^{\prime}\right)=\left(a-a^{\prime}\right) \circ\left(a-a^{\prime}\right)=\left|a-a^{\prime}\right|^{2}>0 .
$$

(Of course if all of $A$ lies on one point, and all of $B$ lies on a second point we would have to resort to another motion.)

This type of motion will work for a sphere in any dimension. In a very real sense this example represents the essential background for all of Theorem 1. If we take projective maps, these motions can be transfered to 
any ellipsoid, hyperboloid of two sheets, or elliptical paraboloid in space. In this projective theory the velocities are expressed as weighted sections of a plane (or hyperplane in $n$-space) normal to the velocity $[3,8]$. If we have these velocities recorded as sections of the tangent plane to the sphere at the joint, then the projected image will be sections of the tangent planes to the new quadric at the joints (with the weight changed by the projection). Back in Euclidean terms the velocities will be appropriately scaled normals to the new quadric surface.

In real 3-space, the hyperboloids of one sheet are not projectively equivalent to the sphere we have discussed. However all the quadric surfaces are projectively equivalent in complex projective space. When we move to complex space, we keep the linear algebra $((v-v) \circ(a-b)=0)$ but abandon any reference to preserving distances: thus our complex "sphere" has equation $Z \circ Z=r(\operatorname{not} Z \circ \bar{Z}=r)$. With this choice, all of our previous comments continue to apply, and the hyperboloid is also the projective image of the sphere, and has the corresponding infinitesimal motion with velocities normal to the surface. However in this case there is a possible flaw in the proof that the motion is internal on the points of the framework (when the two joints lie on a line in the surface). We will clarify this situation in Examples 2 and 3.

To cover the degenerate quadric surfaces (cones, cylinders, two planes) we can express the surface as the limit of non-degenerate surfaces and locate the velocities from the limit. There is some room for confusion in the special case of two planes, one containing $A$, the second containing $B$. Here the limit of internal motions normal to the surface seems to turn into a rigid motion, although we anticipate 3 degrees of internal freedom. We will clarify this situation in Example 4, using algebra. However it is a general theorem that the limit of frameworks with internal motions must have an internal motion, so we really have given a geometric proof for Corollary 1.1 .

The special case of Theorem 1 in which $A$ lies on a plane conic with $B$ in space seems out of place in this geometry. However the points $A$, together with tangent planes to the conic normal to the plane of $A$, share a quadric surface with any chosen $b$ in space. If we take the velocities from this surface, the common tangent planes at $A$ will ensure a uniform choice of velocities for $A$ while each $b$ moves in its own separate world (Figure 3).

For those who are comfortable with projective geometry, and the representation of velocities by hyperplanes, this informal discussion, together with the direct arguments in Case 4 of Theorem 1, does explain all of the motions which we have detected. However a detailed presentation 
of the algebra may clarify the situation and will provide the clues needed for the other results in the paper. Accordingly we give an algebraic presentation of the velocities appropriate to quadric surfaces in 3-space. The algebra will also clarify the two difficulties mentioned above.

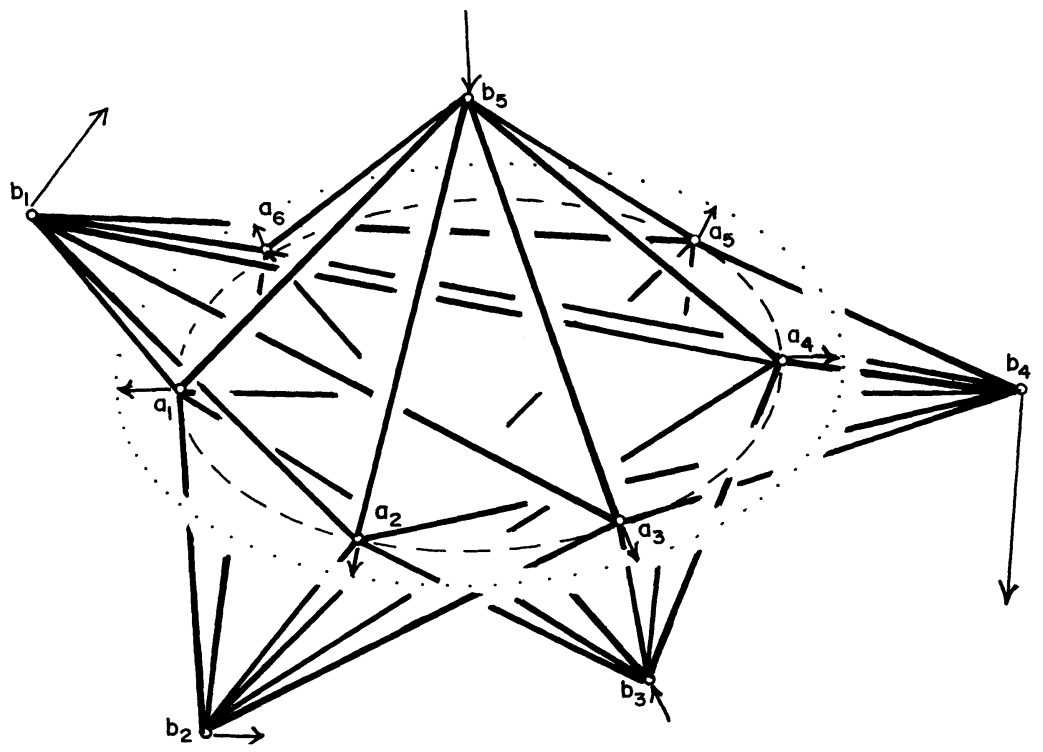

Figure 3

EXAMPLE 2. The non-degenerate ellipsoids and hyperboloids.

By an appropriate choice of coordinates, these surfaces can be expressed in the form

$$
c x^{2}+d y^{2}+e x^{2}+f=0 .
$$

The equation of the tangent plane at $\left(x^{\prime}, y^{\prime}, z^{\prime}\right)$ can be written

$$
\left(c x^{\prime}\right) x+\left(d y^{\prime}\right) y+\left(e z^{\prime}\right) z+f=0 .
$$

We will use the normals to these planes.

For any joint $a=\left(a_{1}, a_{2}, a_{3}\right)$ in $A$ we assign the velocity

$$
u=\left(c a_{1}, d a_{2}, e a_{3}\right) \text {. }
$$

For any joint $b=\left(b_{1}, b_{2}, b_{3}\right)$ in $B$ we assign the velocity

$$
v=-\left(c b_{1}, d b_{2}, e b_{3}\right) \text {. }
$$

It is a simple matter to check that for any bar $a b$ we have the equation

$$
\begin{aligned}
(a-b) \circ(u-v) & =c\left(a_{1}^{2}-b_{1}^{2}\right)+d\left(a_{2}^{2}-b_{2}^{2}\right)+e\left(a_{3}^{2}-b_{3}^{2}\right) \\
& =-f+f=0 .
\end{aligned}
$$

Thus the motion we have defined is permitted. 
Must the motion be internal? Consider two joints $a, a^{\prime}$.

$$
\left(a-a^{\prime}\right)\left(u-u^{\prime}\right)=C\left(a_{1}-d_{1}^{\prime}\right)^{2}+d\left(a_{2}-a_{2}^{\prime}\right)^{2}+e\left(a_{3}-a_{3}^{\prime}\right)^{2}=0 .
$$

For an ellipsoid $(c, d, e>0)$ this will clearly be non-zero, but for some pairs of joints on a hyperboloid it might be zero. When is this possible?

We can get a clearer picture, and include the paraboloids and cones, if we write the quadric surface with the traditional symmetric matrix $Q$, and the point as a column vector $p$. In fact this presentation will cover quadric surfaces in every dimension.

EXAMPLE 3. The general quadric in space. The equation of the surface is $p^{\mathrm{t}} Q p=k$.

For a joint $a \in A$ we assign the velocity $u=a^{\mathrm{t}} Q$, and for a joint in $B$ we assign the velocity $v=-b^{\mathrm{t}} Q$. For any bar $a, b$ we have

$$
(u-v) \circ(a-b)=\left(a^{\mathrm{t}} Q+b^{\mathrm{t}} Q\right) \circ(a-b)=a^{\mathrm{t}} Q a-b^{\mathrm{t}} Q b=0 .
$$

For any pair of joints $a, a^{\prime}$ in $A$ we have

$$
\begin{aligned}
\left(u-u^{\prime}\right) \circ\left(a-a^{\prime}\right) & =\left(a^{\mathrm{t}} Q-a^{\prime \mathrm{t}} Q\right)\left(a-a^{\prime}\right) \\
& =a^{\mathrm{t}} Q a+a^{\prime \mathrm{t}} Q a-a^{\mathrm{t}} Q a^{\prime}-a^{\prime \mathrm{t}} Q a \\
& =2 k-2 a^{\mathrm{t}} Q a^{\prime} .
\end{aligned}
$$

Since $a^{\mathrm{t}} Q a^{\prime}=k$ is the equation of the tangent plane at $a$, we know $a^{\prime}$ is in this tangent plane if the result is 0 . The motion is internal unless $a^{\prime}$ lies in the tangent plane at $a$ and vice-versa. Geometrically this would require that the entire line $a a^{\prime}$ lie in the quadric - as we now confirm algebraically. Consider any point on the line $\lambda a+(1-\lambda) a^{\prime}$ :

$$
\begin{aligned}
(\lambda a & \left.+(1-\lambda) a^{\prime}\right)^{\mathrm{t}} Q\left(\lambda a+(1-\lambda) a^{\prime}\right) \\
& =\lambda^{2} a^{\mathrm{t}} Q a+(1-\lambda)^{2} a^{\prime \mathrm{t}} Q a^{\prime}+2 \lambda(1-\lambda) a^{\mathrm{t}} Q a^{\prime} \\
& =\lambda^{2} k+(1-\lambda)^{2} k+2 \lambda(1-\lambda) k=k .
\end{aligned}
$$

We conclude that the motion defined by these normals to the quadric surface is internal unless all lines $a_{i} a_{j}$ within $A$ lie in the surface and all lines $b_{i} b_{j}$ within $B$ also lie in the surface. On reflection this leaves one possibility: the joints of $A$ are in one hyperplane and the joints of $B$ are in a second hyperplane (possibly the same hyperplane as $A$ ).

If we have such hyperplanes, with at least one joint on both hyperplanes, then by the types of argument used in Case 4 of Theorem 1, we have an infinitesimal vibration of these points on both planes (the joints are "flat" with all bars in a single hyperplane). 
We will now analyse the basic case in 3-space when no joints lie on the line of intersection.

EXAmple 4. $A$ spans one plane and $B$ spans a second plane (no joints on the line of intersection). By Corollary 1.3 we expect 3 degrees of freedom.

For convenience we will take the projectively general case of two parallel planes $A \subset\{(x, y, 1)\}, B \subset\{(x, y,-1)\}$. We present 3 independent generators for the set of internal motions.

$$
\begin{array}{cl}
M: \text { For } a=(x, y, 1) & \text { assign } u=\left(-2 x, 0, x^{2}\right) . \\
\text { For } b=(x, y,-1) & \text { assign } v=\left(2 x, 0, x^{2}\right) . \\
N: \text { For } a=(x, y, 1) & \text { assign } u=\left(0,-2 y, y^{2}\right) . \\
\text { For } b=(x, y,-1) & \text { assign } v=\left(0,+2 y, y^{2}\right) . \\
L: \text { For } a=(x, y, 1) & \text { assign } u=(-2 y, 0, x y) . \\
\text { For } b=(x, y,-1) & \text { assign } v=(0,2 x, x y) .
\end{array}
$$

In each case the verification that $(a-b) \circ(u-v)=0$ is a simple piece of algebra.

Without loss of projective generality, we can assume that the framework includes the following joints (since $A$ and $B$ span the planes):

$$
\begin{array}{ll}
a_{1}=(0,0,1), & a_{2}=(1,0,1), \quad a_{3}=(s, t, 1), \quad t \neq 0, \\
b_{1}=(0,0,-1), & b_{2}=(0,1,-1),
\end{array}
$$

With these points we can verify the independence of $M, N$ and $L$ in the presence of the rigid motions.

All three motions fix the bar $a_{1} b_{1}$ so the only rigid motion which could possibly be relevant is the rotation giving velocities $R(x, y, z)=$ $(-y, x, 0)$.

Assume $M=\alpha N+\beta L+\gamma R$. Since $N$ and $L$ give zero velocity to $a_{2}$ :

$$
(-2,0,1)=M\left(a_{2}\right)=\overrightarrow{0}+\overrightarrow{0}+\gamma R\left(a_{2}\right)=\gamma(0,1,0) .
$$

We conclude that $M$ is independent of the other motions.

Assume $N=\alpha L+\beta R$. Since $L$ gives zero velocity to $b_{2}$ :

$$
(0,2,1)=N\left(b_{2}\right)=\overrightarrow{0}+\beta R\left(b_{2}\right)=\beta(-1,0,0) .
$$

Thus $N$ is independent of $L$ and $R$. Assume $L=\alpha R$.

$$
(0,0,0)=L\left(a_{2}\right)=\alpha R\left(a_{2}\right)=\alpha(-1,0,0)
$$


and

$$
(-2 t, 0, s t)=L\left(a_{3}\right)=\alpha R\left(a_{3}\right)=\alpha(-t, s, 0) \quad \text { with }-2 t \neq 0 .
$$

Thus $L$ is independent of the rigid motions.

REMARK 1. Where did these motions come from? Consider the two planes as a limit of quadric surfaces through $a_{1}$ and $b_{1}$. We take the motions given by these quadric surfaces, add on the rigid motion which cancels the effects on $a_{1}$ and $b_{1}$, and take the limits of these velocities. This breaks the pattern that velocities are normal to the surface, but gives an internal motion. (Figure 4B.)
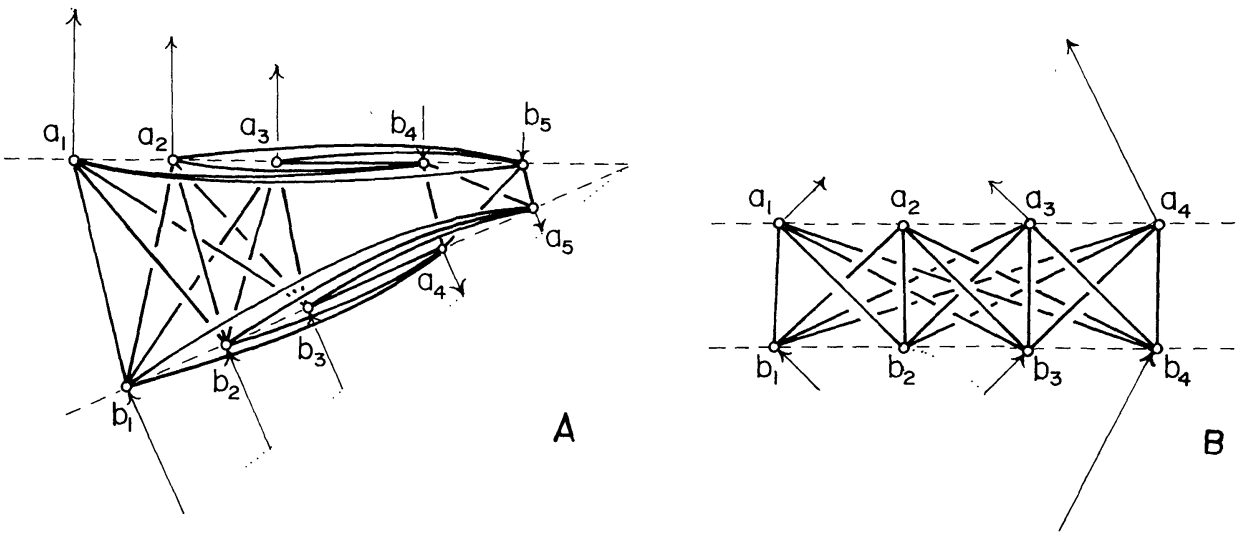

Figure 4

Remark 2. We note that if $A$ and $B$ lie on a degenerate quadric surface of two planes in 3-space with $A$ and $B$ scattered on both planes, the motion does involve the usual normals to the surface (Figure 4A).

REMARK 3. In the plane the case of two hyperplanes becomes two lines and we can use the motion $M$, as given above, in the $x z$ plane. In $d$ space, $d>3$, we expect $\frac{1}{2} d(d-1)$ degrees of internal freedom. The corresponding motions can be obtained by taking analogues of $M$ and $N$ for each of the axis directions except the last, giving $(d-1)$ motions, and an analogue of $L$ for each pair of these directions, giving the remaining $\frac{1}{2}(d-1)(d-2)$ motions to produce the desired basis. 
EXAmple 5. All of $A$ lies in a plane conic (not a single line) and $B$ is scattered throughout space. For simplicity we let the plane be $z=0$, and the equation of the conic be $(x, y)^{t} Q(x, y)=e$. For each $b=\left(b_{1}, b_{2}, b_{3}\right)$, $b_{3} \neq 0$, we use the quadric surface

$$
P(b):(x, y)^{t} Q(x, y)-\frac{\left(\left(b_{1}, b_{2}\right)^{t} Q\left(b_{1}, b_{2}\right)-e\right) z^{2}}{\left(b_{3}\right)^{2}}=e .
$$

All of these surfaces give the same velocities to points in $A$, and the motion on $A$ is an internal motion unless $A$ is collinear. This situation was described in Case 4 of Theorem 1.

REMARK. This technique clearly applies in any dimension. In the plane the lower quadric becomes two points on a line. In 4 space the quadric surface in the hyperplane is a quadric surface in 3-space.

The motions displayed in Examples 3-5 give, along with degeneracies, a lower bound on the internal degree of freedom of the framework. To complete a direct proof of Theorem 1, and the corollaries, we would have to show that these are the only possible internal motions. Provided the number of joints is adequate to span a unique conic, this upper bound can be provided by direct calculations $[5 ; 11]$. However we will continue to rely on the result of Bolker and Roth. In this context our direct geometric display does offer a simplified replacement for the one prolonged calculation in their proof: the demonstration that the presence of a quadric surface through $C$ gives an additional stress [1, Theorem 6].

In closing this presentation of the infinitesimal motions, we want to describe, informally, how the infinitesimal motions effect the calculations of distances in geodesy.

Assume we have $n$ points in 3-space (not all in a plane) with the distances $a_{i} a_{j},(i, j) \in B$, each measured within a small error $\varepsilon^{2}$. If there is a framework, with bar lengths within $\varepsilon^{2}$ of the measured distances, which has an internal infinitesimal motion, then in the calculation of the remaining distances some answers may have an error on the scale of $\varepsilon$ (much larger than $\varepsilon^{2}$ for small $\varepsilon$ ).

Why? Take the framework with the internal motion $v_{i}$ at point $a_{i}$. For some pair of joints (not a bar) $a_{p}, a_{q}$ the internal motion changes the distance:

$$
\left(v_{p}-v_{q}\right) \circ\left(a_{p}-a_{q}\right) \neq 0
$$


If we move along these velocity vectors by $\varepsilon\left(a_{i}^{\prime}=a_{i}+\varepsilon v_{i}\right)$ then the bars have

$$
\varepsilon\left(v_{l}-v_{j}\right) \circ\left(a_{i}-a_{j}\right)=0
$$

so these change length on a scale $\varepsilon^{2}$. For the special pair $a_{p} a_{q}$, the change is on the scale of $\varepsilon$.

If we reverse the motion (taking $-v_{i}$ ) then we reverse the sign of the error, but not the magnitude of the error.

We can use our additional knowledge about the form of the infinitesimal motion of a bipartite framework to understand what combinations of errors are possible near the singular configuration.

We assume, for simplicity, that $A$ moves "outward" and $B$ "inward" from the surface (in the natural sense for ellipsoids extended by projective maps to the other surfaces).

If the critical configuration is a non-degenerate ellipsoid, elliptic paraboloid, or elliptic cylinder, then all "errors" (changes of distance) within $A$ will be positive, and all errors in $B$ will be negative. If the surface is an hyperboloid of two sheets then the errors between points in $A$ on the same sheet remain positive, while errors between pairs on different sheets become negative. This is reversed for joints in $B$.

If the surface is an hyperboloid of 1 sheet or a cone, the "two" lines in the surface, through a joint $a_{1}$, divide the surface into 4 sections. In two of these, the line from $a_{1}$ to $a_{i}$ goes "inside" the surface and this distance is increased by the motion. For the other two sections a line from $a_{1}$ to $a_{j}$ goes outside the surface and the distance is decreased. Of course this description is reversed for joints in $B$.

If the critical configuration is a plane conic through $A$, then there is a finite mechanism giving an almost unlimited error in distance measurements in $A$ and $B$ [13]. Other mechanisms are also possible (among the cases just covered) and these will be presented elsewhere.

If the criticial configuration is two planes, with $A$ in one, $B$ in the other, then a variety of errors is possible, depending on the motion chosen.

Finally we note that this analysis of which distances increase, and which decrease, could also be used to predict which sets of two cables or struts could be added to produce an infinitesimally rigid tensegrity framework [6].

4. Adding bars to a $K_{m, n}$. We will present the discussion in 3-space, although there are no difficulties (other than space and interest) in extending the results to other higher dimensions. 
In describing the infinitesimal motion in Example 2 the one "difficulty" we encountered was that if the line $a_{i} a_{j}$ was on the quadric surface then this distance was not altered. In this case both velocities were normal to the line $a_{i} a_{j}$ - the common line of the tangent planes at $a_{i}$ and $a_{j}$ - and a line in the quadric surface.

We now turn this difficulty into a theorem - such lines in the surface inserted as bars will not remove the infinitesimal motion and any other bar will.

THEOREM 2. A framework realizing $K_{m, n}$ in 3-space ( $\left.m, n \geq 2\right)$ with one added bar $a_{1} a_{2}$ will have an internal motion iff at least one of the following holds:

(i) the joints are contained on a quadric surface containing the line $a_{1} a_{2}$

(ii) the joints of $A$ lie in a plane containing some joint of $B$;

(iii) the joints of $B$ line in a plane containing both $a_{1}$ and $a_{2}$ or containing some other joint of $A$;

(iv) the joints of $B$ lie on a plane conic and the line $a_{1} a_{2}$ touches the conic at 1 point;

(v) the joints of $A$ lie in a plane conic containing the line $a_{1} a_{2}$.

Proof. Once more, we will proceed by cases, depending on the span of $A$ and of $B$.

Case 1. If $m$ or $n \leq 3$ then it is a simple matter to see that adding any single bar leaves an internal motion and leaves a framework satisfying (iv) (if $n \leq 3$ ) or (v) (if $m \leq 3$ ).

Case 2. Both $A$ and $B$ span the space.

(a) If there is only one internal motion in $K_{m, n}$, it comes from a non-degenerate quadric. By Example 3 the bar leaves this motion iff the line of $a_{1} a_{2}$ lies on the quadric surface.

(b) If there is more than one internal motion in $K_{m, n}$, then there is a family of quadric surfaces. One member of this family also includes the point $\frac{1}{2} a_{1}+\frac{1}{2} a_{2}$ and thus the whole line. The framework always has an internal motion and satisfies (i).

Case 3. $A$ spans a plane with some joint $b$ on this plane. The internal motion which vibrates $b$ vertical to this plane always remains, and the framework satisfies (ii). 
Case 4. $A$ spans a plane, $B$ spans a second plane. By Corollary 1.3 there are at least 3 degrees of internal freedom in the $K_{m, n}$. The added bar will always lie in the degenerate quadric and will block at most one internal motion.

Case 5. $B$ spans a plane and $A$ spans space.

(a) There is only one internal motion in the $K_{m, n}$.

If this single motion involves a vibration out of the plane by $a_{1}$ or $a_{2}$ then the added bar will stop this motion and the framework lies outside the five conditions.

If the single motion involves the vibration of any other joint $a_{i}$ out of the plane then this motion remains and the framework satisfies (iii).

If the single motion involves a conic through $B$ (with no joints of $A$ in the plane) then we will use the description of the motion presented in Example 5. Without loss of projective generality we can assume the line $a_{1} a_{2}$ is normal to the plane of the conic: $a_{1}=(p, q, r), a_{2}=(p, q, s)$ and this line hits the plane at $(p, q, 0)$. If we write $(p, q)^{t} Q(p, q)=f$ then the joints have the following velocities:

$$
\begin{aligned}
& \text { For } a_{1}: u_{1}=\left((p, q)^{t} Q, \frac{f-e}{r^{2}} \circ r\right) \\
& \text { For } a_{2}: u_{2}=\left((p, q)^{t} Q, \frac{f-e}{s^{2}} \circ s\right) .
\end{aligned}
$$

Since the joints have the same $x$ and $y$ components of velocity, the problem of preserving the length $\left|a_{1}-a_{2}\right|$ reduces to examining $(r-s)(f-e)$ $\circ(1 / r-1 / s)$. If the line $a_{1} a_{2}$ touches the conic then $f=e$ and length is preserved. Otherwise the given equation requires $r=s$, which was assumed false ( $a_{1} a_{2}$ is a bar!). Thus condition (iv) of the statement is accurate.

(b) There is more than 1 internal motion in the $K_{m, n}$. The bar must leave an internal motion. We now check that the framework will also satisfy one of conditions (iii) or (iv).

If at least two motions involve vibrating joints $a_{i}, a_{j}$ then condition (iii) must be satisfied.

If any joint other than $a_{1}$ or $a_{2}$ lies in the plane, condition (iii) is also satisfied.

By Corollary 1.4, we can now assume that at most one joint $a_{1}$ or $a_{2}$ lies in the plane, and if it does it also lies on a conic through $B$. Such a joint gives a line $a_{1} a_{2}$ touching the conic satisfying condition (iv). Otherwise since there is more than 1 degree of internal freedom and no joints of 
$A$ lie in the plane, the joints of $B$ lie on a family of conics. One of this family will include the point of intersection of $a_{1} a_{2}$ with the plane, satisfying condition (iv).

Case 6. $A$ is collinear, $|B|>3$. Adding one bar will leave at least $|B|-1$ internal motions (by Corollary 1.5 ) and the framework satisfies condition (v).

Case 7. $B$ is collinear and $|A|>3$. Adding the one bar will leave at least $|A|-2$ motions (by Corollary 1.5), and the framework will have a degenerate plane conic including the line of $B$ and say joint $a_{1}$, satisfying condition (iv).

The statement and proof were long because we were examining a large variety of bipartite frameworks. If we take a single bipartite framework $K_{4,5}$, with the correct size to just be infinitesimally rigid when we add one bar, then we regain a desirable simplicity. This example was examined in $[5$, p. 70$]$.

COROLlaRY 2.1 A framework in 3-space with the graph $K_{4,5}$ plus any single bar has an internal motion iff there is a quadric surface through the nine joints which contains the line of the added bar or the four joints $a_{1} a_{2} a_{3} a_{4}$ are coplanar.

Proof. Clearly both configurations described can be fitted into the conditions of Theorem 5 , noting that when $a_{1} a_{2} a_{3} a_{4}$ are coplanar we can find a conic containing any other desired point.

We leave it to the reader to verify that any framework satisfying Theorem 2 will also satisfy these simpler conditions.

We now inquire about bipartite frameworks with many bars added. It is tempting to hope that we could just rewrite Theorem 2 with a larger number of bars added. However we could view a tetrahedron as a $K_{1,3}$ with three bars $b_{1} b_{2}, b_{2} b_{3}$, and $b_{3} b_{1}$ added and it is rigid, in spite of lying on a ruled quadric. Even adding 2 more joints to make a $K_{3,3}$ plus three bars will not help.

Rather than be exhaustive (and exhausting ) about all the cases at hand we will offer necessary and sufficient conditions for internal motions in several non-degenerate cases. 
THEOREM 3. Assume a framework in 3-space has a graph: $K_{m, n}$ plus added edges $C \subseteq A \times A$ and $D \subseteq B \times B$, and there are no flat joints ( joints with all entering bars in a single plane).

(i) If $A$ and $B$ span space, there is an internal motion iff there is a quadric surface containing all the joints and all the lines of bars in $C \cup D$.

(ii) If $A$ spans a plane and $B$ spans a plane, and no joints lie on the intersection of the two planes, then there is an internal motion iff there are two points $p$ and $q$ on the (projective) intersection of the two planes such that each line of a bar in $C \cup D$ passes through one of these points.

(iii) If $A$ spans a plane and $B$ spans space, with $B^{\prime}=\bar{A} \cap B$ then there is an internal motion iff there is a conic in the plane containing all joints of $B^{\prime} \cup A$ and all bars of $D \cap\left(B^{\prime} \times B^{\prime}\right)$ as well as of $C$, and this conic touches the line of any other bar in $D$.

Proof. Case 1. If $A$ and $B$ span space, then by Corollary 1.2 and Example 3 all internal motions reflect quadrics. There will be an internal motion remaining iff one of these quadric surfaces contains all the added bars.

Case 2. $A$ spans a plane and $B$ spans a second plane, with no joints on the line of intersection.

We can take the projectively general case of Example 4, and assume that $p$ and $q$ are the points at infinity in the directions of the $x$ - and $y$-axes.

Any bar through $p$ will leave motions $N$ and $L$ of Example 4 and will eliminate any component of the motion $M$.

Similarly, any bar through $q$ will eliminate any component of the notion $N$, while leaving $M$ and $L$.

If both kinds of bars are present then the one remaining internal motion is type $L$. If a further bar which is not through $p$ or $q$ is added then $L$ is also blocked (since $L$ changes instantaneously the length of any other bar).

We conclude that there is an internal motion iff the required points $p$ and $q$ exist.

Case 3. $A$ spans a plane and $B$ spans space, with $B^{\prime}=\bar{A} \cap B$. Corollary 1.4 says that the degree of internal freedom is $\left|B^{\prime}\right|+q\left(A \cup B^{\prime}\right)$. Since no joint is flat, we know that each $b_{l} \in B^{\prime}$ has an added bar into space which blocks its vibration out of the plane, eliminating the $\left|B^{\prime}\right|$ degrees of internal freedom, and leaving only the motions due to $q\left(A \cup B^{\prime}\right)$. 
To these motions generated by the conics we apply the argument used in Case 5 (a) of Theorem 2. The derived motion of a conic will be blocked by any bar $b_{i} b_{j}$ missing the conic or by any new bar in the plane which does not lie on the conic. It is now clear that there is an internal motion iff some conic avoids both pitfalls.

REMARK. We can extend part (ii) to include joints on the line of intersection of the planes, once more requiring that $p$ or $q$ lie on the line of any added bars. However the proof would require either a discussion of motions of points at infinity (see [8, §9]), or a new calculation of $M, N$ and $L$ after a projective transformation. We have resisted both alternatives.

However we can make our assertion plausible by viewing the two planes as the limit of a series of ruled hyperboloids with the pencils of lines through $p$ and $q$ as the limit of the rulings (Figure 5). The internal motions on the hyperboloids will pass to the required internal motion on the two planes. The remark following Corollary 1.4 also adds to the plausibility that two points $p$ and $q$ are necessary.

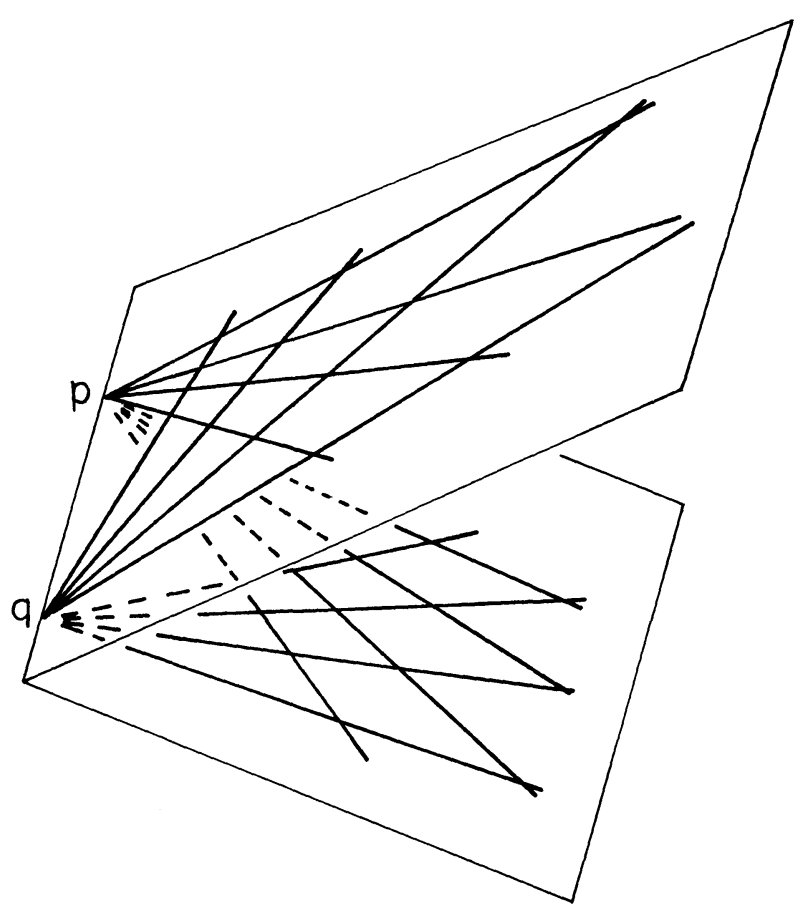

FIGURE 5. Limit of ruled hyperboloids 
Theorem 3 will also simplify if we take a framework which has just enough bars to be infinitesimally rigid in some realization in space $(|E|=3|V|-6)$. Some cases of this were observed in [5, p. 70].

COROLlaRY 3.1. A framework in space with the underlying graph $K_{4,4}$ plus any two additional bars has an internal motion iff there is a quadric surface through all of the joints and containing the lines of the two added bars.

Proof. It is clear that such a quadric surface will usually yield an internal motion. Degenerate positions, as the limit of more general positions on the quadric, must also have an internal motion. We leave it to the reader to verify that for this graph the conditions in Theorem 3, as well as all other degeneracies, lead to the required quadric surface.

5. An application to space frames. The frameworks we have analyzed seem very abstract and impractical as engineered buildings. It is hard to visualize any engineer building a $K_{m, n}$ in space to enclose a room or support a building. We do not know of any built structure with a large $K_{m, n}$ inside. However there is a very standard engineering structure which is a subset of the kind of frameworks analyzed in $\$ 4-$ the tetrahedral/half-octahedral truss (Figure 6) [2].

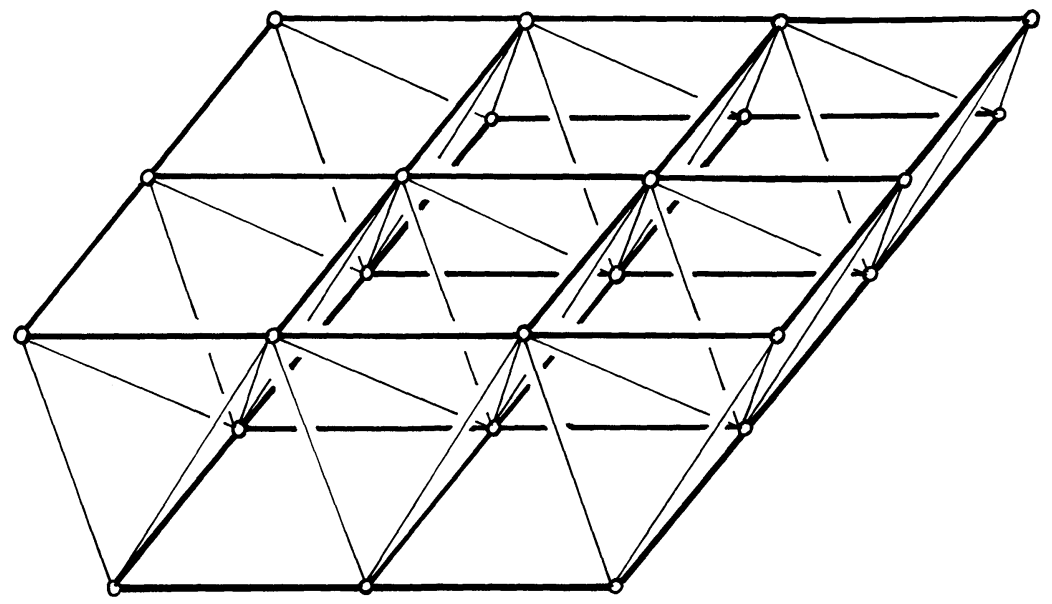

Figure 6 
Most standard trusses begin with two sets of joints ( $A$ and $B$ ) in two parallel planes and a set of bars connecting the two planes (a subset of $\left.K_{A, B}\right)$. In the tetrahedral half-octahedral truss, the additional bars form two parallel square grids, one in each plane. While this graph, even with only part of $K_{A, B}$, is correct to be infinitesimally rigid in most realizations in space, as built it is known to have exactly 1 internal motion [2].

Drawing on Theorem 3 (ii), we see that the truss is contained in the limiting case of a ruled hyperboloid. Therefore even a complete $K_{A, B}$ would not have eliminated the remaining internal motion. If you build a model of a section of this grid, and let it move, then you will observe the characteristic properties which we have predicted. (With the play of the joints the infinitesimal motion becomes a small finite motion.) The distance between any pair of points on the top and bottom layers remains constant. The rulings (the lines of the grids) remain straight. The top and bottom planes each warp into a ruled hyperboloid - two parallel images of the limiting surfaces which explained the motion. When the grid assumes this characteristic shape of a warped bedspring, further infinitesimal motion is blocked.

What can we learn from this explanation? The difficulty with the grid is not in the regular spacings of the two grids, nor in the symmetric staggering of the two layers, nor in the bars chosen to join the two planes. To make an infinitesimally rigid space frame we must either:

(i) use 3 directions for our bars in the planes, or

(ii) break up at least one of the planes.

Since economy and practical construction suggest that we will only use a subset of the $K_{A, B}$ for braces between the planes, these conditions are necessary, but not sufficient for infinitesimal rigidity of a truss.

\section{REFERENCES}

[1] E. D. Bolker and B. Roth, When is a bipartite graph a rigid framework?, Pacific J. Math., 90 (1980), 27-44.

[2] H. Crapo, The tetrahedral/octahedral truss, Structural Topology, 7 (1982), 51-60.

[3] H. Crapo and W. Whiteley, Statics of frameworks and motions of panel structures, Structural Topology, 6 (1982), 43-82.

[4] A. C. Dixon, On certain deformable frameworks, Mess. Math., 29 (1899/1900), 1-21.

[5] K. Killian and P. Meissl, Einige Grundaufgaben der räumlichen Trilateration und ihre gefährlichen Örter, Deutsche Geodätische Komm. Bayer. Akad. Wiss., A61 (1969), 65-72.

[6] B. Roth and W. Whiteley, Tensegrity frameworks, Trans. Amer. Math. Soc., 265 (1981), 419-445. 
[7] W. Whiteley, Motions of bipartite frameworks, Structural Topology, 3 (1979), 62-63.

[8] _ Introduction to structural geometry I: Infinitesimal motions and infinitesimal rigidity, Structural Topology Research Group, U. de Montréal, Montréal, Quebec, preprint.

[9] W. Wunderlich, On deformable nine-bar linkages with six triple joints, Proc. K. Nederl. Akad. Wet., A 79 (1976), 255-262.

[10] __, Gefährliche Annahmen der Trilateration und bewegliche Fachwereke I, Z. Angew. Math. Mech., 57 (1977), 297-304.

[11] __, Gefährliche Annahmen der Trilateration und bewegliche Fachwereke II, Z. Angew. Math. Mech., 57 (1977), 363-368.

[12] Untersuchungen zu einem Trilaterations problem mit komplanaren Standpunkten, Sitz. Osten. Akad. Wiss., 186 (1977), 263-280.

[13] _ Eine merkwürdige Familie von beweglichen Stabwerken, Elem. Math., 34/6 (1979), 132-137.

Received September 24, 1981. This research was supported, in part, by NSERC of Canada Grant A3568 and the manuscript was prepared during a visiting semester at Cornell University.

Champiain Regional College

ST. LAmbert, Quebec, CANADA J4P 3P2

AND

Topologie Structurale

ECOLE D'ARCHITECTURE

UNIVERSITE DE MONTREAL

C.P. 6128 CODE H3C 3J7

Montreal, Quebec, Canada 



\section{PACIFIC JOURNAL OF MATHEMATICS \\ EDITORS}

DONALD BABBITT (Managing Editor)

University of California

Los Angeles, CA 90024

Hugo RossI

University of Utah

Salt Lake City, UT 84112

C. C. MOOre and ARThur OGus

University of California

Berkeley, CA 94720
J. Dugundi

Department of Mathematics

University of Southern California

Los Angeles, CA 90089-1113

R. FINN and H. SAMELSON

Stanford University

Stanford, CA 94305

ASSOCIATE EDITORS

$\begin{array}{lllll}\text { R. Arens } & \text { E. F. Beckenbach } & \text { B. H. Neumann } & \text { F. Wolf } & \text { K. Yoshida }\end{array}$ $(1906-1982)$

\section{SUPPORTING INSTITUTIONS}

UNIVERSITY OF ARIZONA

UNIVERSITY OF BRITISH COLUMBIA

CALIFORNIA INSTITUTE OF TECHNOLOGY

UNIVERSITY OF CALIFORNIA

MONTANA STATE UNIVERSITY

UNIVERSITY OF NEVADA, RENO

NEW MEXICO STATE UNIVERSITY

OREGON STATE UNIVERSITY
UNIVERSITY OF OREGON

UNIVERSITY OF SOUTHERN CALIFORNIA

STANFORD UNIVERSITY

UNIVERSITY OF HAWAII

UNIVERSITY OF TOKYO

UNIVERSITY OF UTAH

WASHINGTON STATE UNIVERSITY

UNIVERSITY OF WASHINGTON 


\section{Pacific Journal of Mathematics}

\section{Vol. 110, No. $1 \quad$ September, 1984}

Wojciech Abramczuk, A class of surjective convolution operators $\ldots \ldots \ldots \ldots 1$

K. Adachi, Extending bounded holomorphic functions from certain

subvarieties of a weakly pseudoconvex domain $\ldots \ldots \ldots \ldots \ldots \ldots$

Malvina Florica Baica, An algorithm in a complex field and its application

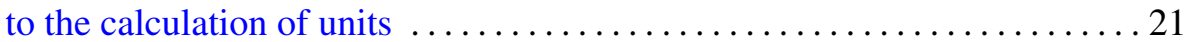

Giuliana Bianchi and Robert Cori, Colorings of hypermaps and a

conjecture of Brenner and Lyndon $\ldots \ldots \ldots \ldots \ldots \ldots \ldots \ldots \ldots \ldots \ldots \ldots$

Ronald James Evans, Determinations of Jacobsthal sums . . .......... 49

Leslie Foged, Characterizations of $\aleph$-spaces .................... 59

Nassif A. Ghoussoub and Paulette Saab, Weak compactness in spaces of

Bochner integrable functions and the Radon-Nikodým property . . . . . . 65

J. Gómez Gil, On local convexity of bounded weak topologies on Banach

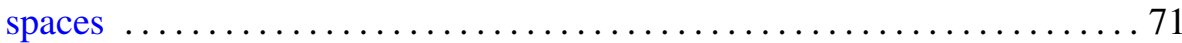

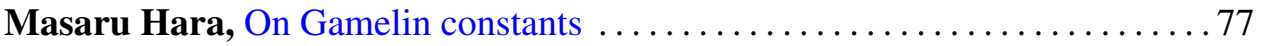

Wilfried Hauenschild, Eberhard Kaniuth and Ajay Kumar, Harmonic

analysis on central hypergroups and induced representations $\ldots \ldots \ldots 83$

Eugenio Hernandez, An interpolation theorem for analytic families of

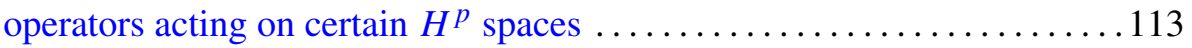

Thomas Alan Keagy, On "Tauberian theorems via block-dominated

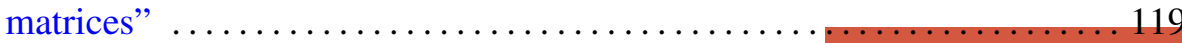

Thomas Landes, Permanence properties of normal structure $\ldots \ldots \ldots \ldots \ldots 125$

Daniel Henry Luecking, Closed ranged restriction operators on weighted

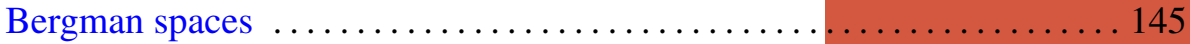

Albert Thomas Lundell, The $p$-equivalence of $\mathrm{SO}(2 n+1)$ and $\mathrm{Sp}(n) \ldots \ldots 161$

Mark D. Meyerson, Remarks on Fenn's "the table theorem" and Zaks' "the chair theorem" ..................................... 167

Marvin Victor Mielke, Homotopically trivial toposes . . . . . . . . . . 171

Gerard J. Murphy, Hyperinvariant subspaces and the topology on Lat A . . 183

Subhashis Nag, On the holomorphy of maps from a complex to a real manifold

Edgar Milan Palmer and Robert William Robinson, Enumeration of self-dual configurations ................................. 203

John J. Walsh and David Clifford Wilson, Continuous decompositions

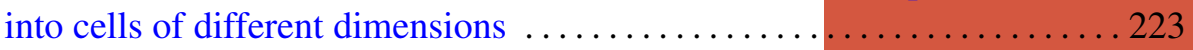

Walter John Whiteley, Infinitesimal motions of a bipartite framework .....233 